\title{
Photoelectron holography of the $\mathrm{H}_{2}^{+}$molecule
}

\author{
Gellért Zsolt Kiss ${ }^{1,2,3, a}$, Sándor Borbély ${ }^{3}$, Attila Tóth ${ }^{4}$, and Ladislau Nagy ${ }^{3}$ \\ 1 Wigner Research Centre for Physics, Budapest H-1121, Hungary \\ 2 National Institute for Research and Development of Isotopic and Molecular Technologies, Donat 61-103, Cluj-Napoca \\ Ro-400293, Romania \\ 3 Faculty of Physics, Babeş-Bolyai University, Kogălniceanu 1, Cluj-Napoca Ro-400084, Romania \\ ${ }^{4}$ ELI-ALPS, ELI-HU Non-profit Ltd., Dugonics tér 13, Szeged H-6720, Hungary
}

Received 30 May 2019 / Received in final form 28 February 2020

Published online 18 June 2020

(c) The Author(s) 2020. This article is published with open access at Springerlink.com

\begin{abstract}
We investigate the photoelectron spectrum of the $\mathrm{H}_{2}^{+}$target induced by few-cycle XUV laser pulses using first principle calculations. In the photoelectron spectrum, by performing calculations for different internuclear separations, we investigate how the structure of the target is influencing the spatial interference pattern. This interference pattern is created by the coherent superposition of electronic wave packets emitted at the same time, but following different paths. We find that the location of the interference minima in the spectra is dominantly determined by the target's ionization energy, however, by comparing the $\mathrm{H}_{2}^{+}$results with model calculations with spherically symmetric potentials, clear differences were observed for the molecular potential relative to the central potentials. Next to the main feature (spatial interference) we have also identified the traces of the two-center interference in the photoelectron spectrum, however, these were mainly washed out due to the complex electronic wave packet dynamics that occurs during the interaction with the considered laser field.
\end{abstract}

\section{Introduction}

As in the case of its optical analogy [1], the electron holography [2] captures both the phase and amplitude information of the electron wave packets (EWPs) scattered by the target, which is achieved by the interference between the scattered and a reference wave. In traditional electron holography the electron wave packets are created by the electron gun of an electron microscope and manipulated by imaging elements $[2,3]$. In contrast, in photoelectron holography the EWPs are created via the ionization of the target by an intense ultrashort laser pulse, and are manipulated by the electric field of the same laser pulse. Compared to the traditional electron holography, photoelectron holography is a relatively new technique. The first experimental observation of a photoelectron hologram [4] dates back only a few years, and in order to become a mature experimental technique, a detailed understanding of the underlying processes is required.

The formation of the photoelectron holograms can also be interpreted as a secondary process following the primary ionization of a target induced by an ultrashort laser pulse, which modulates the photoelectron momentum distribution. These modulations are the results of the superposition of electronic wave packets created at the same time (i.e., during the same quarter-cycle of the pulse),

\footnotetext{
${ }^{a}$ e-mail: zsolt.kiss@itim-cj.ro
}

but which follow different spatial paths. This is one of the several possible EWP interference scenarios [5], and it is also known as spatial interference. In the case of spatial interference, along the different spatial paths each wave packet accumulates a different phase, which due to the coherent superposition leads to the formation of a radial ridge structure in the electron spectra [4-14]. In the framework of a simplified two-path model [4-6] the spatial interference pattern can be understood as the result of the interference between the direct (i.e., weakly scattered by the parent ion) and indirect (i.e., strongly scattered) EWPs. The existence of these two distinct wave packets was confirmed by an elaborate classical trajectory MonteCarlo simulation [10] for the hydrogen target, where it was shown that electrons can reach a given continuum state with a well defined momentum by following two types of trajectories: weakly scattered by the core (i.e., the minimum distance between the returning electron and the parent ion is larger than 5 a.u.), or strongly scattered ones (i.e., the minimum distance between the returning electron and the parent ion is $\sim 1$ a.u.). In this picture, the weakly scattered waves can be considered as part of the reference wave packet, while the strongly scattered EWPs as part of the signal wave packet, which confirms the interpretation of the spatial interference pattern as the hologram of the target $[4,10]$.

Both the photoelectron holography and the laserinduced electron diffraction (LIED) [15-19] rely on the 
scattering by the parent ion of the laser field induced EWPs during their quiver motion. The main difference between the two is that in the case of LIED the reference wave is missing (i.e., the continuum EWP created during the ionization step is not broad enough in the momentum space). The transition between the photoelectron holography and LIED is gradual, and it can be achieved by narrowing the continuum electron wave packet in momentum space in the direction perpendicular to the laser polarization axis. From the photoelectron momentum distribution resulted from this electronic wave packet diffraction both structural [15-18] and temporal [19] information regarding the target atom or molecule can be extracted using laborious procedures [17]. In photoelectron holography, in addition to the LIED's scattered electronic wave packet (signal) a reference wave packet is also present, and the coherent superposition of these two EWPs leads to a more structured electron momentum distribution with clearly identifiable interference minima and maxima. This, in principle, allows for an easier extraction of structural information from a photoelectron hologram, than from a LIED pattern.

Due to previous experimental $[4,6,8,11]$ and theoretical $[10,12-14,20,21]$ investigations several aspects of the formation of the photoelectron holograms are understood. Firstly, for a given target, the density of the interference minima is determined by the $z_{0}$ parameter, which measures the maximum distance from the parent ion reached by the EWPs before the scattering event. The value of $z_{0}$ is directly controlled by the parameters of the driving laser pulse, and its increase can be achieved by increasing the pulse intensity or wavelength. Secondly, it was recently shown [13] that in the case of atomic targets for a fixed driving laser pulse the shape of the hologram is strongly influenced by the scattering potential (i.e., the atomic species of the target). This high target sensitivity of the photoelectron holography is due to the fact that the phase accumulated by the scattered EWP is strongly influenced by the depth of the binding potential in the vicinity of the target core. In order to continue this line of inquiry, in the present paper, we investigate how the photoelectron hologram looks like for molecular targets, and how the geometry of the molecular target is influencing the obtained hologram. We chose the $\mathrm{H}_{2}^{+}$molecular ion as our target $[22,23]$, and we solved numerically the time-dependent Schrödinger equation (TDSE) considering different internuclear distances. In order to highlight the multi-center effects of the molecular binding potential on the hologram we also performed calculation for two different model $\mathrm{H}_{2}^{+}$targets. In the first model we have substituted the two-center binding potential of $\mathrm{H}_{2}^{+}$with a one center potential obtained by averaging over the molecular axis orientation $\left(\mathrm{H}_{2 \bmod 1}^{+}\right)$, while in the second case $\left(\mathrm{H}_{2 \bmod 2}^{+}\right)$we have used a modified version of the model potential from [24].

Several studies [25-34] on the photoelectron holography of molecular targets were already performed, which, with the exception of a few studies [26,27,31,32], were focusing on the $\mathrm{H}_{2}^{+}$target. A significant portion of these works $[26,27,29,34]$ investigated the influence of the molecular axis orientation on the hologram at equilibrium internuclear distance, and found that the forward scattering photoelectron hologram is only weakly influenced by it. This weak effect was explained by the observation that in the case of the small molecules the electron scattering cross section in the forward scattering direction is mainly determined by the long-range Coulomb potential $[32,35]$, and the short-range effects are repressed by the long range contribution. The molecular axis orientation dependence of the forward scattering photoelectron hologram can be investigated by increasing the molecular axis length $[30,33]$ or by the use of circularly polarized driving laser field $[28,29]$. For the backward scattering direction the contribution of the short-range part of the molecular potential is larger, thus as it was expected the backward scattering photoelectron hologram (the signal and reference wave packets being created during different quarter cycles $[25,32]$ ) is much strongly influenced by the molecular axis orientation [32]. In contrast, investigations on the molecular axis length dependence of the forward scattering photoelectron hologram are sparse. In [32] the indirect experimental evidence is presented on the molecular axis length dependence of the photoelectron hologram. Furthermore, in the framework of a simplified model, where the $\mathrm{H}_{2}^{+}$was described by a 2D soft-core Coulomb potential, this effect is explicitly investigated $[30,33]$. In the first study [30] it is only marginally discussed, while in the second study [33] it is investigated in detail only at large internuclear distances (in the region of the chargeresonance enhanced ionization).

In the above outlined context, the present study is dedicated to the investigation of the molecular axis length dependence of the forward scattering photoelectron hologram (photoelectron hologram in the forthcoming part of the paper), where special attention will be accorded to the physics of the molecular axis length dependence of the photoelectron hologram. Furthermore, for the equilibrium internuclear distance, the laser field intensity dependence of the photoelectron hologram will also be analyzed in order to check the validity of previous observations [10] made for atomic targets.

The present paper is structured as follows: the introduction is followed by the theory section, where our approach for the numerical solution of the TDSE for the $\mathrm{H}_{2}^{+}$targets is outlined. This is followed by a section dedicated to the performed numerical convergence tests. Thereafter we present and discuss our results obtained for the $\mathrm{H}_{2}^{+}$target followed by a comparison to the $\mathrm{H}_{2 \bmod 1}^{+}$and $\mathrm{H}_{2 \bmod 2}^{+}$model targets. The last section is dedicated to the conclusions. Throughout the present article atomic units are used.

\section{Numerical solution of the TDSE for the $\mathrm{H}_{2}^{+}$ molecule}

During our investigations we have considered the interaction between few-cycle XUV laser pulses and the $\mathrm{H}_{2}^{+}$molecule. For such ultrashort laser fields the BornOppenheimer approximation can be safely used, thus the electronic and nuclear dynamics can be separated. 

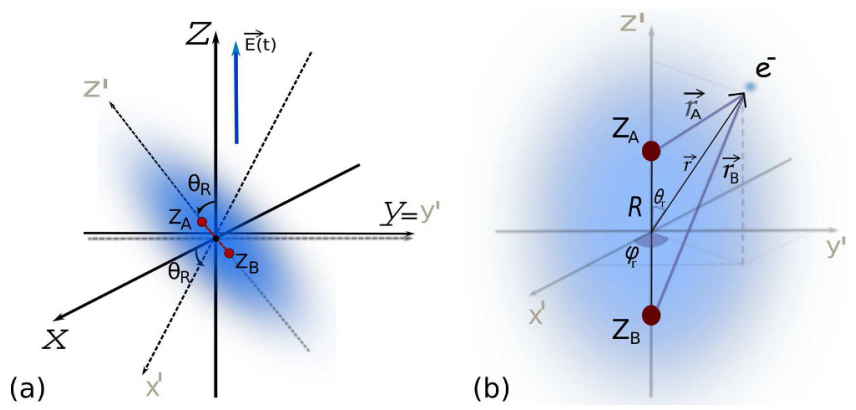

Fig. 1. The geometry of the studied system in the laboratory (a) and in the molecular frame (b). The $x^{\prime} y^{\prime} z^{\prime}$ molecular frame is rotated by the angle $\theta_{R}$ around the $0 y=0 y^{\prime}$ axis. The electric component of the incident laser field is linearly polarized in the $0 z$ direction.

Moreover, the fixed nuclei approximation was considered since the vibrational period of the target is much larger than the duration of the driving field, thus we restricted ourselves to the solution of the electronic TDSE for fixed internuclear separation

$$
i \frac{\partial}{\partial t} \Psi(\boldsymbol{r} ; t)=\left[\hat{H}_{0}+\hat{U}_{\mathrm{int}}(t)\right] \Psi(\boldsymbol{r} ; t)
$$

where the time-dependent electronic Hamiltonian was given as the sum of the $\hat{H}_{0}=\hat{T}+\hat{V}$ field-free and the $\hat{U}_{\text {int }}(t)=\boldsymbol{r} \cdot \boldsymbol{E}(t)$ electron-laser interaction term written in the dipole approximation using the length gauge. $\hat{T}$ is the kinetic energy operator of the electron with mass $\mu$ at position $\boldsymbol{r}$, while $\hat{V}=-Z_{\mathrm{A}} / r_{\mathrm{A}}-Z_{\mathrm{B}} / r_{\mathrm{B}}$ represents the Coulomb interaction potential between the electron and the two nuclei located at distances $r_{\mathrm{A}, \mathrm{B}}$ from the electron. The electric charges of the nuclei were set to $Z_{\mathrm{A}}=Z_{\mathrm{B}}=1$ $\left(\mathrm{H}_{2}^{+}\right)$. In our calculations we employed linearly polarized laser fields in the $O z$ direction (which defines the laboratory frame) having a sine-square envelope:

$$
E(t)= \begin{cases}E_{0} \sin \left(\omega t+\varphi_{\mathrm{CEP}}\right) \sin ^{2}\left(\frac{\pi t}{\tau}\right), & \text { if } t \in[0, \tau] \\ 0, & \text { otherwise }\end{cases}
$$

where $\omega$ is the carrier frequency, $\tau$ is the pulse duration, $\varphi_{\mathrm{CEP}}$ is the carrier-envelope-phase, and $E_{0}$ represents the amplitude of the electric field. Figure 1 illustrates the geometry of the studied system in the laboratory and the molecular frame of reference.

Both the $\Psi(t, \boldsymbol{r})=\sum_{m=-\infty}^{\infty} \Psi^{(m)}(\xi, \eta ; t) e^{i m \varphi} / \sqrt{2 \pi}$ $(m \in \mathbb{Z})$ wave function $(\mathrm{WF})$ and the Hamiltonian were expressed in the prolate spheroidal coordinate system: $\xi=\left(r_{\mathrm{A}}+r_{\mathrm{B}}\right) / R, \eta=\left(r_{\mathrm{A}}-r_{\mathrm{B}}\right) / R, \varphi$ (the azimuthal angle); with $R$ being the internuclear distance, and $\xi \in[1, \infty)$, $\eta \in[-1,1], \varphi \in[0,2 \pi][36-42]$. Then, they were represented on finite-element discrete variable representation (FE-DVR) grids [43-46] (for both $\xi$ and $\eta$ configuration subspaces), where the local basis functions [47] were built using rescaled Lagrange interpolation polynomials (DVR functions) built on top of the $x_{i}^{\alpha}\left(w_{i}^{\alpha}\right)$ Gauss-Lobatto quadrature points (weights).
Moreover, we employed a rescaled form of the WF

$$
\Psi^{(m)}\left(\xi_{i}, \eta_{j} ; t\right)=\psi_{i j}^{(m)}(t) / \sqrt{\left(R^{3} / 8\right) w_{i}^{\{\xi\}} w_{j}^{\{\eta\}} J\left(\xi_{i}, \eta_{j}\right)},
$$

with $J\left(\xi_{i}, \eta_{j}\right)=J_{i j}=\xi_{i}^{2}-\eta_{j}^{2}$ being the Jacobian. By using this ansatz the TDSE in the FEDVR basis takes the following form

$$
i \frac{\partial \psi_{i j}^{(m)}(t)}{\partial t}=\sum_{m^{\prime} i^{\prime} j^{\prime}}\left[T_{i j i^{\prime} j^{\prime}}^{m m^{\prime}}+V_{i j i^{\prime} j^{\prime}}^{m m^{\prime}}+U_{i j i^{\prime} j^{\prime}}^{m m^{\prime}}(t)\right] \psi_{i^{\prime} j^{\prime}}^{\left(m^{\prime}\right)}(t),
$$

where the coupling (Hamiltonian) matrix elements $H_{i j i^{\prime} j^{\prime}}^{m m^{\prime}}(t)=T_{i j i^{\prime} j^{\prime}}^{m m^{\prime}}+V_{i j i^{\prime} j^{\prime}}^{m m^{\prime}}+U_{i j i^{\prime} j^{\prime}}^{m m^{\prime}}(t)$ were decomposed to

$$
\begin{aligned}
T_{i j i^{\prime} j^{\prime}}^{m m^{\prime}}= & 2 \delta_{m m^{\prime}} \mu^{-1} R^{-2}\left[\delta_{i i^{\prime}} \delta_{j j^{\prime}} m^{2}\left(\xi_{i}^{2}-1\right)^{-1}\right. \\
& \times\left(1-\eta_{j}^{2}\right)^{-1}+\left(\delta_{j j^{\prime}}\left\langle f_{i}\left|\hat{T}_{\xi}\right| f_{i^{\prime}}\right\rangle\right. \\
& \left.\left.+\delta_{i i^{\prime}}\left\langle g_{j}\left|\hat{T}_{\eta}\right| g_{j^{\prime}}\right\rangle\right) J_{i j}^{-1 / 2} J_{i^{\prime} j^{\prime}}^{-1 / 2}\right] \\
V_{i j i^{\prime} j^{\prime}}^{m m^{\prime}}= & \delta_{m m^{\prime}} \delta_{i i^{\prime}} \delta_{j j^{\prime}} V\left(\xi_{i}, \eta_{j}\right) \\
U_{i j j^{\prime} j^{\prime}}^{m m^{\prime}}(t)= & {\left[2 \delta_{m m^{\prime}} \xi_{j} \eta_{j} \cos \theta_{R}+\left(\delta_{m-1, m^{\prime}}+\delta_{m+1, m^{\prime}}\right)\right.} \\
& \left.\times \sqrt{\left(\xi_{i}^{2}-1\right)\left(1-\eta_{j}^{2}\right)} \sin \theta_{R}\right] \delta_{i i^{\prime}} \delta_{j j^{\prime}} E(t) R / 4
\end{aligned}
$$

It is worth mentioning, that in the case when the molecular axis is parallel to the laser polarization vector, the coupling between the partial channels corresponding to different $m$ values vanishes, since the second term in equation (7) is zero for $\theta_{R}=0$.

In order to obtain a symmetric form for the kinetic energy matrix $T_{i j i^{\prime} j^{\prime}}^{m m^{\prime}}$, the matrix elements of the $\hat{T}_{\xi}$ and $\hat{T}_{\eta}$ operators were symmetrized by following the procedures outlined in $[37,38]$ :

$$
\begin{aligned}
& \left\langle f_{i}\left|\hat{T}_{\xi}\right| f_{i^{\prime}}\right\rangle \simeq \sum_{k} w_{k}^{\{\xi\}}\left(\xi_{k}^{2}-1\right) f_{i}^{\prime}\left(\xi_{k}\right) f_{i^{\prime}}^{\prime}\left(\xi_{k}\right) . \\
& \left\langle g_{j}\left|\hat{T}_{\eta}\right| g_{j^{\prime}}\right\rangle \simeq \sum_{l} w_{l}^{\{\eta\}}\left(1-\eta_{l}^{2}\right) g_{j}^{\prime}\left(\eta_{l}\right) g_{j^{\prime}}^{\prime}\left(\eta_{l}\right),
\end{aligned}
$$

where $f_{i}(\xi)$ and $g_{j}(\eta)$ are the rescaled Lagrange interpolation polynomials built on top of the $\xi$ and $\eta$ grid.

Since, in the present work the orientation of the molecular axis was constrained parallel to the laser field polarization vector $\left(\theta_{R}=0\right)$ and due to the cylindrical symmetry around the molecule, the coupling between the partial channels corresponding to different $m$ values disappeared. Starting the simulation from the $m=$ $0\left|1 s \sigma_{g}\right\rangle$ ground state the dimensionality of the original $3 \mathrm{D}$ problem was reduced to $2 \mathrm{D}$. This initial WF (and the other higher lying bound states used during the calculation of the photoelectron spectra) was obtained for fixed internuclear distances via the direct diagonalization of the field-free Hamiltonian using the Scalable Library for Eigenvalue Problem Computations (SLEPc) package [48]. Afterwards, this initial WF was propagated in time using 
the short-iterative Lanczos (SIL) scheme [49], where in each fixed-size $\left(\Delta t=10^{-3}\right.$ a.u. $)$ time step the propagation error was kept below $10^{-8}$ by automatically adapting the size of the Krylov subspace in which the evolution operator was evaluated.

\subsection{Numerical details}

In order to avoid the undesired reflections from the edge of the simulation grid at $\xi_{\max }$, we have utilized a complex absorbing potential of the form:

$$
V_{\mathrm{CAP}}=-i \exp \left[\alpha_{\mathrm{abs}} \log \cos \frac{\xi-\xi_{\mathrm{cut}}}{\xi_{\max }-\xi_{\mathrm{cut}}}\right] \text {, for } \xi \geq \xi_{\mathrm{cut}},
$$

where the value of $\alpha_{\text {abs }}$ was set to $10^{6}$ and $\xi_{\text {cut }}$ at $0.95 \xi_{\max }$.

The proper size of our $\xi$ simulation box was ensured by keeping the norm of the absorbed part of the WF below $10^{-10}$. For the results presented in this paper this condition was always fulfilled with the simulation box-size $\xi_{\max }=1220$ a.u. $/ R$. The number of DVR basis functions for both the $\xi$ and $\eta$ grids was set to $N_{\text {fun }}=N_{\text {fun }}^{\{\xi\}}=$ $N_{\text {fun }}^{\{\eta\}}=7$. For all internuclear separations the size of the $\eta$ FEs was fixed to $\Delta_{\eta}=10^{-1}$ (resulting in an average $\eta$ gridpoint distance of $\left.\Delta_{\eta} /\left(N_{\text {fun }}^{\{\eta\}}-1\right) \simeq 1.6 \times 10^{-2}\right)$, while the size of the $\xi$ FEs varied as a function of $R$ in such a way that $\Delta_{\xi} R$ was kept constant at 2.4 a.u. This translates to $121 \eta$ and $3073 \xi$ gridpoints leading to the Hamiltonian matrix size of $371833 \times 371833$. With these grid parameters the calculated bound state energies were in excellent agreement with the data found in [41], e.g., the relative differences between the two sets of data for the first $10 \mathrm{BS}$ energies for $R=2 \mathrm{a} . \mathrm{u}$. were below $10^{-10}$. These grid parameters also ensured that even electronic states with high momentum and angular momentum were properly represented.

\section{Calculation of convergent photoelectron spectra}

The most straightforward way of obtaining the ionization probability density [i.e., the photoelectron spectrum $(\mathrm{PhES})]$ is by projecting the propagated WF onto the exact scattering states $\left|\Psi_{\boldsymbol{k}}\right\rangle$ with asymptotic electron momentum $\boldsymbol{k}$

$$
\frac{\mathrm{d} P}{\mathrm{~d} \boldsymbol{k}}(\boldsymbol{k} ; t)=\left|\left\langle\Psi_{\boldsymbol{k}} \mid \Psi(t)\right\rangle\right|^{2} .
$$

Considering that the two-center continuum WFs do not have analytical expressions - there are only laborious numerical procedures [50] to obtain them - we restricted our calculations to one-center continuum functions. Since these scattering states are approximate for the $\mathrm{H}_{2}^{+}$molecule they introduce a certain amount of error in the calculated electron spectra. The first source of error lies in the fact that the one-center functions are not orthogonal to the bound state (BS) wave functions of $\mathrm{H}_{2}^{+}$.

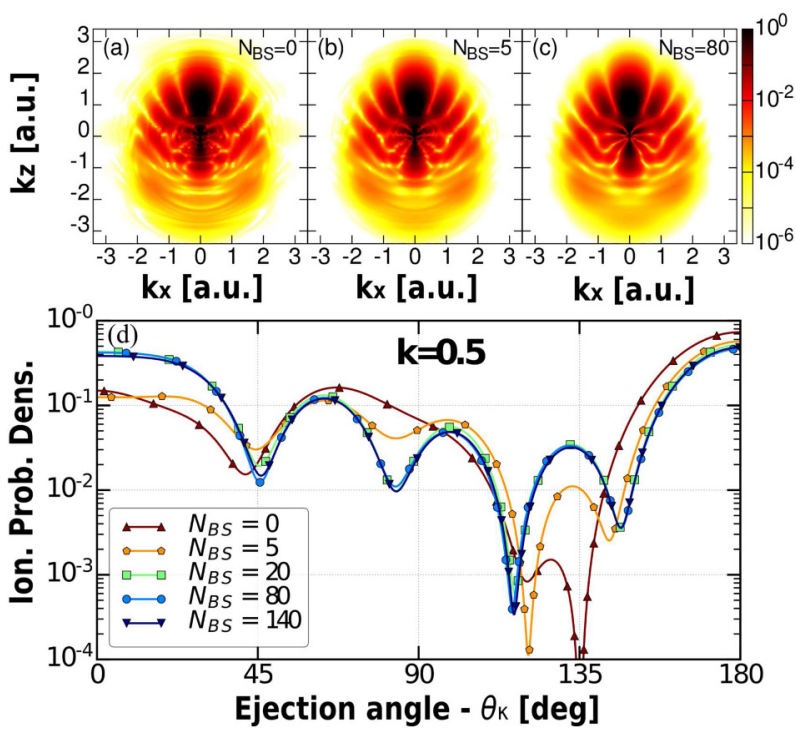

Fig. 2. Convergence of the photoelectron spectra calculated as a function of the number of subtracted bound states $\left(N_{\mathrm{BS}}\right)$ calculated at time moment $5 \tau$ for the internuclear separation $R=4$ a.u.: (a) $N_{\mathrm{BS}}=0$, (b) $N_{\mathrm{BS}}=5$, (c) $N_{\mathrm{BS}}=80 . k_{x}$ is the electron momentum component perpendicular, while $k_{z}$ parallel to the laser polarization vector. In (d) the ionization probability density at $t=5 \tau$ for the fixed $k=\sqrt{k_{x}^{2}+k_{z}^{2}}=$ 0.5 a.u. momentum is shown as a function of $N_{\mathrm{BS}}$.

This source of error can be eliminated by removing the bound part of the time-dependent WF prior to projecting it onto the continuum states [Eq. (11)]. The second source of error is rooted in the fact that while in the asymptotic region the one- and two-center Coulomb wave functions are similar, in the close vicinity of the nuclei they differ significantly. This error can be greatly reduced by propagating the TDSE until the continuum part of the WF departs sufficiently far from the vicinity of the nuclei.

Throughout this work we used a two-cycle XUV laser pulse [see Eq. (2)] with $\omega=0.4445$ a.u., $\tau=28.27$ a.u., $E_{0}=0.5$ a.u., and $\varphi_{\mathrm{CEP}}=-(\omega \tau+\pi) / 2$ (resulting in a symmetric pulse in time), and calculated the photoelectron spectra for different internuclear distances: $R=1$, 2 , and 4 a.u. As mentioned above the use of one-center continuum WFs introduces potentially two types of error. This section is dedicated to show that these errors can be reduced to minimum. First, we investigated how the number of the subtracted bound states influences the PhES, second how these spectra depend on the propagation time after the end of the laser pulse. We performed the convergence tests for all considered internuclear distances, and found a similar behavior for all $R$ values. Here we show the detailed results for $R=4$ a.u.

\subsection{Convergence of the bound state subtraction}

At time moment $5 \tau$ (measured from the start of the laser pulse) we calculated the PhES for cases when a different number of BSs $\left(N_{\mathrm{BS}} \in\{0,5,20,80,140\}\right)$ were subtracted from the TDWF. These results are shown in Figure 2. 
The striking differences between the PhESs are the concentric rings appearing at low momenta for small $N_{\mathrm{BS}}$ values (Figs. 2a and $2 \mathrm{~b}$ ). These rings are the direct consequence of the non-orthogonality of the $\mathrm{H}_{2}^{+} \mathrm{BSs}$ with respect to the used approximate scattering states. The projection of the bound part of the TDWF onto single center Coulomb wave functions is non-vanishing, and during the calculation of the PhES it is coherently added to the projection of the continuum part of the TDWF leading to the observed concentric ring structures. Increasing the number of subtracted BSs these rings fade away, i.e., for $N_{\mathrm{BS}}=5$ they are already significantly reduced, while for $N_{\text {BS }} \geq 20$ they were barely visible. This behavior is also observable in Figure 2d, where the PhES is presented as a function of electron ejection angle (measured from the polarization vector of the laser field) for a fixed electron momentum $k=0.5 \mathrm{a} . \mathrm{u}$. We see a significant change in the PhES as $N_{\text {BS }}$ is increased from 0 to 5, and then to 20, but for $N_{\mathrm{BS}} \geq 20$ the changes in the PhES are negligible. After analyzing the spectrum at different fixed electron momentum values, by scanning both the lower and higher momentum parts of the PhES, we found no significant differences between the results obtained for $N_{\mathrm{BS}} \geq 20$.

A similar behavior was observed for the other $R$ values, however with the decrease in $R$ the number of subtracted states required for a converged PhES increased. This is explained by the larger ionization potentials associated with smaller internuclear distances, which results in a smaller amount of continuum WF, i.e., a larger portion of the WF remains distributed among the BSs. Accordingly, the results presented in this article were calculated for $N_{\mathrm{BS}}=120$.

\subsection{Convergence as a function of propagation time}

In Figures 3a and 3b the PhESs calculated for $R=4 \mathrm{a} . \mathrm{u}$. at $t \in\{\tau, 5 \tau\}$ are shown. For an easier comparison, Figure 3c presents segments of the PhES for the same $R$ at a fixed electron ejection angle $\theta_{k}=40^{\circ}$ (i.e., the approximate direction of the first maximum in Figs. 3a and $3 \mathrm{~b})$ for $t \in\{\tau, 2 \tau, \ldots, 6 \tau\}$.

It can be seen that these spectra are mostly sensitive to changes in the final propagation time at small momentum values. While a noticeable change can be observed for low electron momentum ( $k \leq 1$ a.u.), in the higher momenta region changes are barely visible and they disappear quickly after the conclusion of the oscillating field. This behavior can be again explained by the difference between the exact scattering states of $\mathrm{H}_{2}^{+}$and the approximate one-center functions employed in this work, which is the largest in the immediate vicinity of the target. The continuum EWPs corresponding to the high momentum part ( $k \geq 1$ a.u.) of the PhES depart quickly from the immediate vicinity of the target, and for $t \simeq 2 \tau$ they already reach the region of the coordinate space where the differences between the two types of continuum functions become insignificant. In contrast, for the low momentum part of the PhES ( $k \leq 1$ a.u.) a "full" convergence is not achieved even when $t=6 \tau$ (see the zoomed inset in Fig. 3c), since the corresponding continuum EWPs
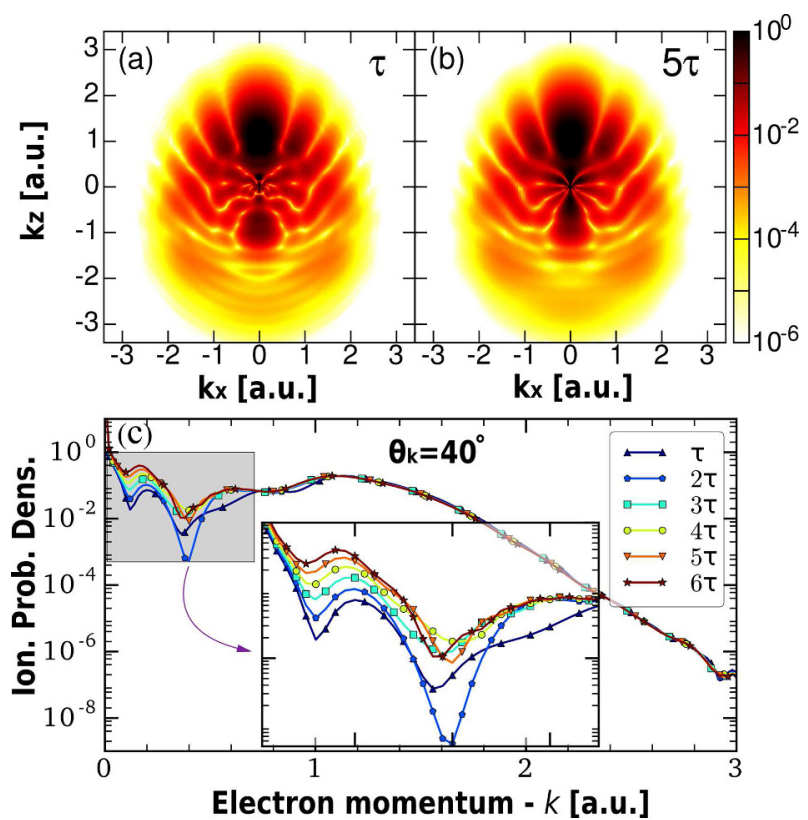

Fig. 3. Ionization probability density calculated for the internuclear distance $R=4 \mathrm{a} . \mathrm{u}$. as a function of electron momentum component perpendicular $\left(k_{x}\right)$ and parallel $\left(k_{z}\right)$ to the laser polarization vector calculated at time moments: (a) $\tau$; (b) $5 \tau$; (c) segments of the electron spectra at different time moments for the fixed ejection angle $\theta_{k}=\tan ^{-1}\left(k_{x} / k_{z}\right)=40^{\circ}$ in the range of $k \in[0,3]$ is shown, while in the inset a zoom into the range of $k \in[0,1]$ can be seen.

are departing much slower from the close vicinity of the molecule.

A "full" convergence (for small $k$ values as well) can be achieved by propagating the WF even further in time. However, for those calculations the coordinate space simulation box should be increased as well (to prevent absorption and reflection at the simulation box boundary), which would imply a non-negligible increase in the CPU time required for the simulations. In order to quantitatively describe the convergence of the spectra the

$$
\mathcal{E}^{R}(t)=\frac{\iint \mathrm{d} k_{x} \mathrm{~d} k_{z}\left|P\left(k_{x}, k_{z} ; t\right)-P\left(k_{x}, k_{z} ; \tau\right)\right|}{\iint \mathrm{d} k_{x} \mathrm{~d} k_{z} P\left(k_{x}, k_{z} ; \tau\right)}
$$

quantity was introduced, which measures the relative difference between the PhES calculated at the end of the laser pulse $(t=\tau)$ and the spectra calculated at a later time moment $t>\tau$. It was observed for all considered $R$ values that $\mathcal{E}^{R}(t)$ exponentially converged towards an asymptotic value $\mathcal{E}_{\infty}^{R}$, which was obtained by fitting the calculated $\mathcal{E}^{R}(t)$ data points with the function $\mathcal{E}_{\text {fit }}^{R}(t)=$ $\mathcal{E}_{\infty}^{R}-\beta e^{-\alpha t}\left(\alpha, \beta \in \mathbb{R}^{+}\right)$, and the relative error of the PhES was estimated as:

$$
\delta_{\text {conv }}^{R}(t)=\mathcal{E}_{\infty}^{R}-\mathcal{E}^{R}(t)
$$

In Figure 4 this error estimate was plotted as a function of time along with its exponential fit $\left(\beta e^{-\alpha t}\right)$ for the different internuclear distances. For each internuclear separation $\delta_{\text {conv }}^{R}(t)$ showed an excellent agreement with 


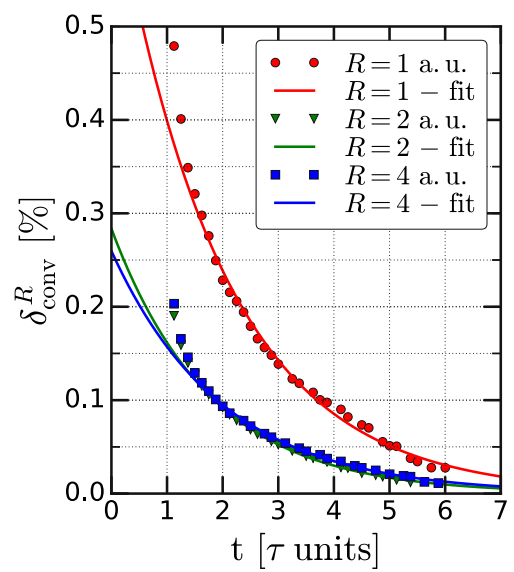

Fig. 4. The error estimate $\delta_{\text {conv }}^{R}$ as a function of time for different $R$ values. The data points are shown along with the $\beta e^{-\alpha t}$ fit.

the used exponential fit confirming the assumed exponential decrease in the projection error. As it is indicated in Figure 4, for the propagation time $5 \tau$ the error estimate in the case of each $R$ is below $0.5 \%$. Since this remaining error is affecting only the low momentum part of the PhES, and the dominant features of the holograms are located at the higher momentum parts of the spectra, for the results presented in the following, the time propagation was ended at $t=5 \tau$.

\section{Results}

The main goal of the present work is to investigate the structure of the photoelectron holograms for molecular targets, i.e., how the geometry of the molecule is influencing the hologram. To this end we present here PhES obtained for the $\mathrm{H}_{2}^{+}$target with different internuclear separations interacting with a few-cycle laser pulse $\left[\omega=0.4445\right.$ a.u., $\tau=28.27$ a.u., $E_{0}=0.5$ a.u., $\varphi_{\mathrm{CEP}}=$ $-(\omega \tau+\pi) / 2]$. Based on previous $[10,13,20,21]$ studies we know that the shape of the hologram is predominantly influenced by two factors. The first one is the spatial path of the strongly scattered (signal) electron trajectory $[10,20,21]$, which is characterized by the $z_{0}$ parameter meaning the maximum distance reached by the continuum EWP before the rescattering event. The second factor is the potential experienced in the immediate vicinity of the target by the returning electron along the signal trajectory [13]. In order to identify the influence of the molecular binding potential on the photoelectron hologram, we have also performed calculations for two model systems with spherically symmetric potentials, which have the same asymptotic forms as the $\mathrm{H}_{2}^{+}$target, and when they interact with a few-cycle laser field they produce similar signal EWP trajectories with the $\mathrm{H}_{2}^{+}$target, i.e., they have the same $z_{0}$ parameter. This second condition can be fulfilled by ensuring that the ionization energy of the model potentials and the $\mathrm{H}_{2}^{+}$target is the same [13]. If these two conditions are met, than the difference observed in the PhES can be directly attributed to the difference
Table 1. The values of the equivalent internuclear distances used for the $\mathrm{H}_{2 \bmod 1}^{+}$(second column), and of the $\alpha$ parameter used for the $\mathrm{H}_{2 \bmod 2}^{+}$model target system (third column).

\begin{tabular}{lcc}
\hline$R\left(\mathrm{H}_{2}^{+}\right)[$a.u.] & $R_{\text {equiv }}\left(\mathrm{H}_{2 \bmod 1}^{+}\right)[$a.u. & $\alpha\left(\mathrm{H}_{2 \mathrm{mod} 2}^{+}\right)\left[\right.$a.u. $\left.^{2}\right]$ \\
\hline 1.0 & 0.92 & -0.18322 \\
2.0 & 1.72 & -0.73734 \\
4.0 & 3.01 & -2.56654 \\
\hline
\end{tabular}

between the binding potentials in the immediate vicinity of the targets [13].

The first model potential was constructed by performing the molecular axis orientation averaging of the potential created by the two nuclei of $\mathrm{H}_{2}^{+}$, which leads to the following form

$$
V_{\bmod 1}(r)= \begin{cases}-2 / r, & \text { if } r \geq R / 2 \\ -4 / R, & \text { if } r<R / 2\end{cases}
$$

with $R$ being the internuclear distance of $\mathrm{H}_{2}^{+}$. Compared to $\mathrm{H}_{2}^{+}$the ionization energy of this model system is lower (the deep potential well around the cores disappears as a result of the orientation averaging), thus, in order to ensure the same ionization energy, the model potential should be modified by performing the following substitution $R \rightarrow R_{\text {equiv }}$, where $R_{\text {equiv }}$ is a model parameter. The value of $R_{\text {equiv }}$ for each internuclear separation is listed in Table 1 (second column).

For the second model potential we have used a modified version of the potential found in [24]

$$
V_{\bmod 2}(r)=-\frac{2}{r}\left\{1+\frac{\alpha}{|\alpha|} \exp \left[-\frac{2 r}{|\alpha|^{1 / 2}}\right]\right\},
$$

and for each internuclear distance we set the value of the model parameter $\alpha$ such, that the ground state energy of the model system reproduced the ground state energy of the $\mathrm{H}_{2}^{+}$target. These values of $\alpha$ are listed in Table 1 (third column).

By comparing the PhES obtained for the $\mathrm{H}_{2}^{+}$(first row in Fig. 5) target and for its models $\left(\mathrm{H}_{2 \bmod 1}^{+}\right.$, second row; $\mathrm{H}_{2 \bmod 2}^{+}$, third row in Fig. 5), at first sight one can observe that the $\mathrm{H}_{2}^{+}$and its model counterpart results for the corresponding $R$ are qualitatively similar. The similarities between the holograms obtained for the $\mathrm{H}_{2}^{+}$and for its corresponding models is not surprising, since the model targets were constructed in such a way that their ionization potentials coincide. Thus, under the action of the same driving laser field for all targets the direct and scattered paths are roughly the same, which ensures that the phases accumulated by the electron along these paths are similar for all targets. The agreement between the PhESs obtained for the different model potentials is very good, differences between the two models are barely observable only for $R=4$ a.u. (see the panels Figs. $5 \mathrm{f}$ and $5 \mathrm{i}$ ). This good agreement appears because the two model potentials are very similar with the exception of the $r<R / 2$ vicinity of the target. The differences between the $\mathrm{H}_{2}^{+}$and model 


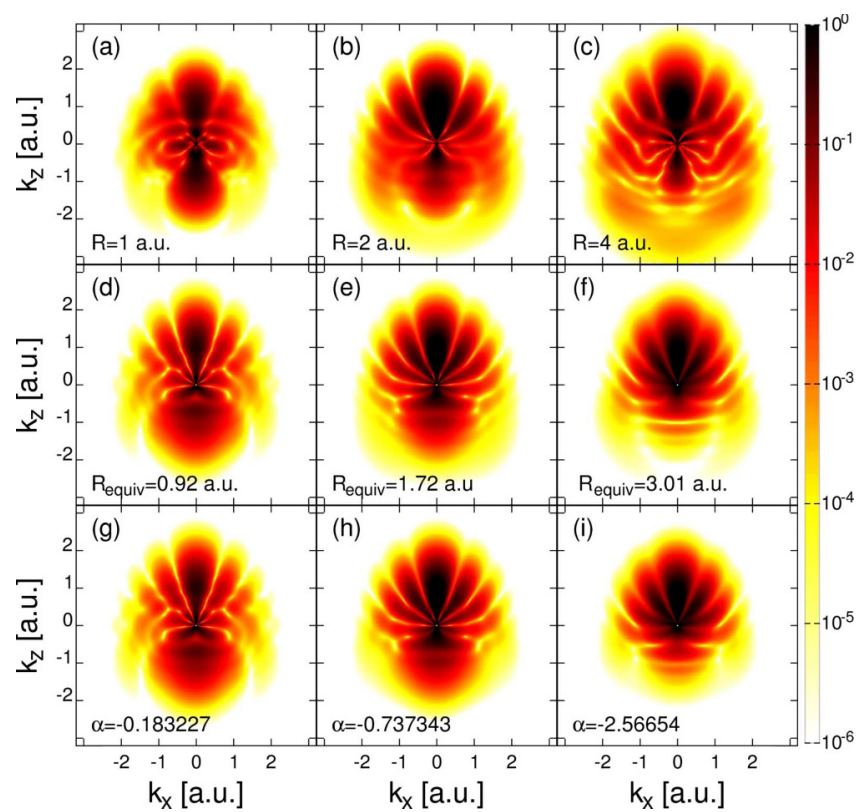

Fig. 5. The converged photoelectron spectra calculated for the $\mathrm{H}_{2}^{+}$(first row) are shown for different internuclear distances: (a) $R=1$ a.u., (b) $R=2$ a.u., (c) $R=4$ a.u. The $\mathrm{H}_{2 \bmod 1}^{+}[(\mathrm{d})$, (e) and (f)] and $\mathrm{H}_{2 \bmod 2}^{+}[(\mathrm{g})$, (h) and (i)] results corresponding to each internuclear separation are shown below the $\mathrm{H}_{2}^{+}$. For a given $R$ the ionization energy of the $\mathrm{H}_{2}^{+}$and the model targets is the same: $I_{p}^{(a)}=I_{p}^{(d)}=I_{p}^{(g)} ; I_{p}^{(b)}=I_{p}^{(e)}=I_{p}^{(h)} ; I_{p}^{(c)}=I_{p}^{(f)}=$ $I_{p}^{(i)}$.

results, however, are directly attributed to the fact that in a larger neighborhood of the targets the binding potentials differ. This difference is illustrated in Figure 6, where $\rho\left[V(\rho, z)-V_{\bmod 1}(\rho, z)\right]$ is shown in the $\rho O z$ plain. Here $\rho=\sqrt{x^{2}+y^{2}}$ denotes the cylindrical coordinate.

The discrepancies between the holograms obtained for $\mathrm{H}_{2}^{+}$and for its models can be further investigated by comparing the angular distribution of photoelectrons (see Fig. 7$)$ at a fixed electron momentum value ( $k=1.5$ a.u.) for different internuclear separations. For each internuclear distance in the forward electron ejection direction $\left(\theta_{k}<90^{\circ}\right)$ the deep minima associated with the spatial interference can be clearly identified for both the $\mathrm{H}_{2}^{+}$ target and for its models. Moreover, the electron ejection angles at which these interference minima appear roughly coincide for all targets. However, in the case of the model targets, regardless of the internuclear distance, the interference minima are systematically located at slightly smaller electron ejection angles, which translates to a slightly denser hologram (i.e., smaller angular separation between the interference minima). The denser hologram in the case of the model systems can be directly attributed to the fact that the returning electron along a large portion of the scattered trajectory meets a deeper binding potential [13]. This is confirmed in Figure 6, where it can be observed, that with the notable exception of the immediate vicinity of the $\mathrm{H}_{2}^{+}$cores $\rho\left[V(\rho, z)-V_{\bmod 1}(\rho, z)\right] \geq 0$. Figure 7 also shows that for the forward electron scattering $\left(\theta_{k}<90^{\circ}\right)$ the difference between the two model

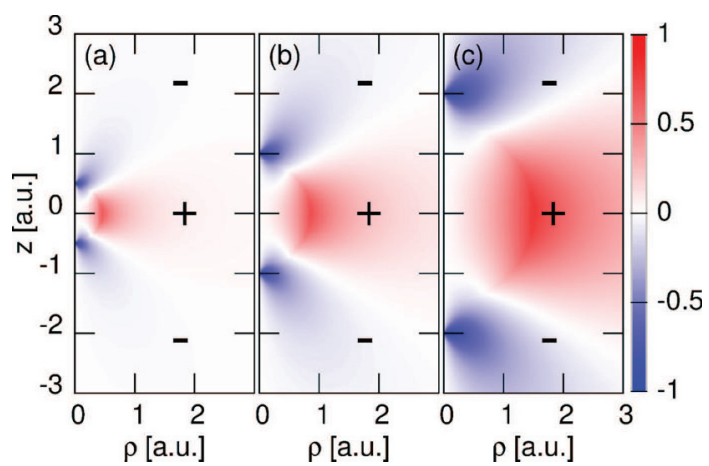

Fig. 6. The value of $\rho\left[V(\rho, z)-V_{\bmod 1}(\rho, z)\right]$, where $\rho$ and $z$ are the cylindrical coordinates, calculated for the corresponding pairs: (a) $R\left(\mathrm{H}_{2}^{+}\right)=1$ a.u., $R_{\text {equiv }}\left(\mathrm{H}_{2 \bmod 1}^{+}\right)=0.92$ a.u.; (b) $R\left(\mathrm{H}_{2}^{+}\right)=2$ a.u., $R_{\text {equiv }}\left(\mathrm{H}_{2 \bmod 1}^{+}\right)=1.72$ a.u.; (c) $R\left(\mathrm{H}_{2}^{+}\right)=$ 4 a.u., $R_{\text {equiv }}\left(\mathrm{H}_{2 \bmod 1}^{+}\right)=3.01$ a.u.

calculations is small even for the largest internuclear separation. In contrast, for the backward electron scattering $\left(\theta_{k}>90^{\circ}\right)$ these differences are much larger since the backward scattering can be attributed to electron trajectories with smaller impact parameters, which map the region of the coordinate space where the difference between the two model potentials is the largest. This is in agreement with the observation [32,35] that the electron scattering cross section in the forward direction is mainly influenced by the long-range part of the binding potential, while in the backward direction by the short range part.

Based on the above arguments, it is clear, that the observed differences between the holograms of the real $\mathrm{H}_{2}^{+}$ target and its models can be attributed to the difference between the two-center binding potential of $\mathrm{H}_{2}^{+}$and the central binding potential of the model targets.

Nonetheless, obvious traces of the two-center interference [51-53] in the hologram of the $\mathrm{H}_{2}^{+}$target are not observed at first sight. This might be due to the fact that the two dominant continuum electronic wave packets are created during the second and the third half cycle $[10,13]$ of the driving laser pulse during a relatively large duration of time (compared to the laser field period). Each of these dominant continuum EWPs can be decomposed into smaller continuum wave packets, which are created over a short period of time, in which the vector potential of the laser field can be considered constant. In these smaller EWPs the two-center interference pattern is present as it is illustrated in Figure 8, where the EWP obtained for a short half-cycle electric pulse corresponding to a small segment of the driving laser pulse is shown in momentum space. As a consequence of the continuing action of the driving electric field, these small EWPs (along with the attached two-center interference patterns) will be shifted in momentum space in accordance with the vector potential value in the moment of their creation. After the completion of the laser pulse, when these wave packets are coherently added, the interference pattern is averaged out since the two-center interference pattern of each smaller EWP is shifted in momentum space with a different value. 


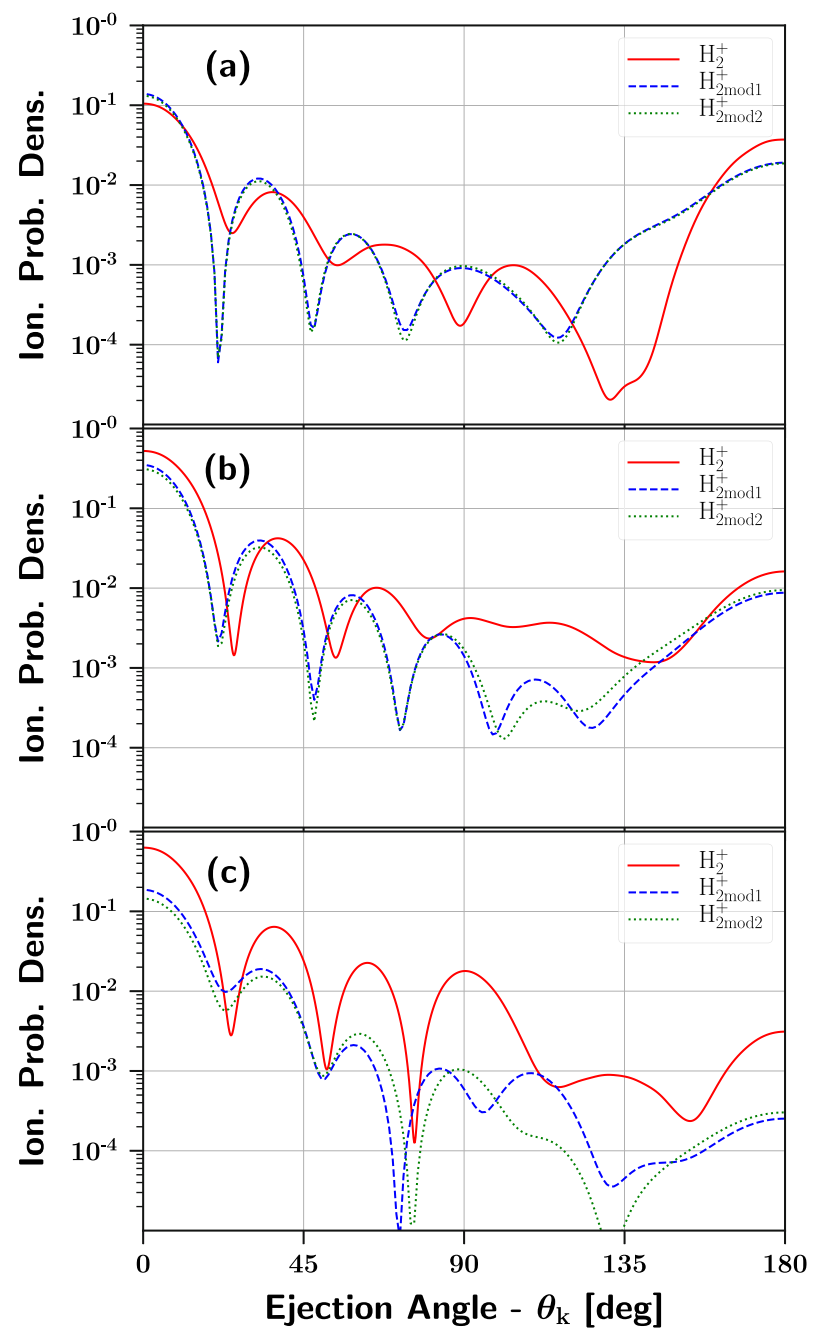

Fig. 7. The angular distribution of photoelectrons at the fixed $k=1.5$ a.u. momentum value for the different targets: (a) $R\left(\mathrm{H}_{2}^{+}\right)=1$ a.u.; (b) $R\left(\mathrm{H}_{2}^{+}\right)=2$ a.u.; (c) $R\left(\mathrm{H}_{2}^{+}\right)=4$ a.u.

For the present laser field parameters, even in the case of the frozen-core approximation, the direct two-center interference minima are smeared out due to the above presented ponderomotive shift effect. This smearing effect may be further amplified if the vibrational motion of the nuclei is included, since the location of the two-center interference minima ${ }^{1}$ shown in Figure 8 strongly depends on the value of the internuclear separation $R$ [54].

The internuclear distance dependence of the photoelectron hologram can be investigated in Figure 5, where it can be observed that by increasing the internuclear distance from 1 a.u. to 4 a.u. the density of the hologram also increased. This behavior can be clearly observed in Figure 9, where the angular distribution of photoelectrons emitted with asymptotic momentum $k=2$ a.u. are shown for different internuclear separations. The number of spatial interference minima in the forward electron ejection region $\left(\theta_{k}<90^{\circ}\right)$ for the $R=1 \mathrm{a}$.u. internuclear

1 Can be approximated by the minima of $\sim \cos ^{2}\left(\frac{\boldsymbol{k} \cdot \boldsymbol{R}}{2}\right)[55]$.

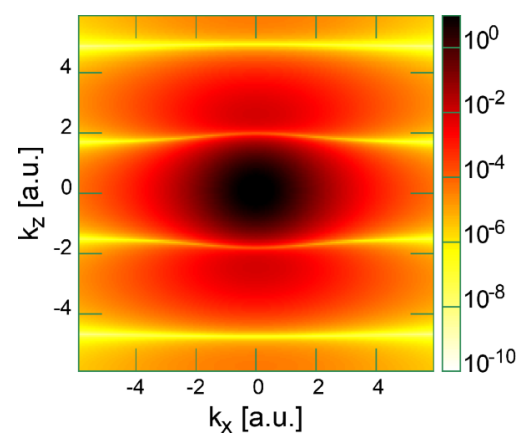

Fig. 8. The EWP in momentum space representation induced on the $\mathrm{H}_{2}^{+}$target by a half-cycle step-like electric pulse with $\tau=0.1$ a.u. duration and $E_{0}=1$ a.u. strength.

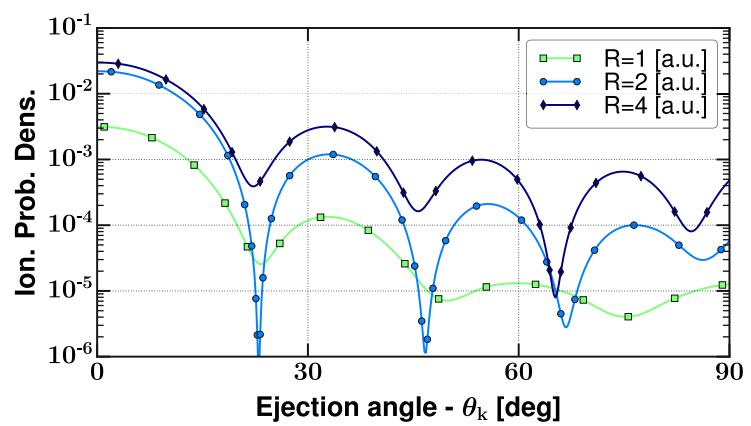

Fig. 9. The $k=2$ a.u. segments of the photoelectron spectra zoomed to the ejection region $\theta_{k}<90^{\circ}$ calculated for the $\mathrm{H}_{2}^{+}$ molecule for the different $R$ internuclear separations.

distance is 3 , which increases to 4 for $R=2$ a.u. and $R=4$ a.u. Moreover, the 4 th interference minimum in the case of $R=4$ a.u. is located at a smaller electron ejection angle than the 4 th interference minimum for $R=2$ a.u. Consequently, the average angular separation between the interference minima decreases, i.e., the density of the interference pattern increases as $R$ gets larger. This increase in the interference pattern's density with increasing internuclear distance is indirectly induced by the drop-off of the ionization energy with the increase in $R$. For a fixed driving laser pulse the decrease in the ionization potential leads to the increase in the initial velocity of the created continuum EWP, which in turn leads to longer electron trajectories prior to the rescattering event. With the increase in the electron trajectory length (i.e., with the increase in the $z_{0}$ parameter) the density of photoelectron hologram increases.

Despite the considerable change of the $\mathrm{H}_{2}^{+}$ionization energy from 1.451 a.u. to 0.796 a.u. when the internuclear distance is increased from 1 to 4 a.u., an anticipated drastic change in the interference pattern's density is not observed. This can be explained by the simple consideration, according to which the expected increase in the interference pattern's density is tempered due to the fact, that with increasing $R$ the depth of the binding potential experienced by the returning electron decreases, which in turn induces a lowering effect in the hologram's density.

For the equilibrium internuclear distance $(R=2$ a.u. $)$ the laser pulse amplitude (intensity) dependence of the 


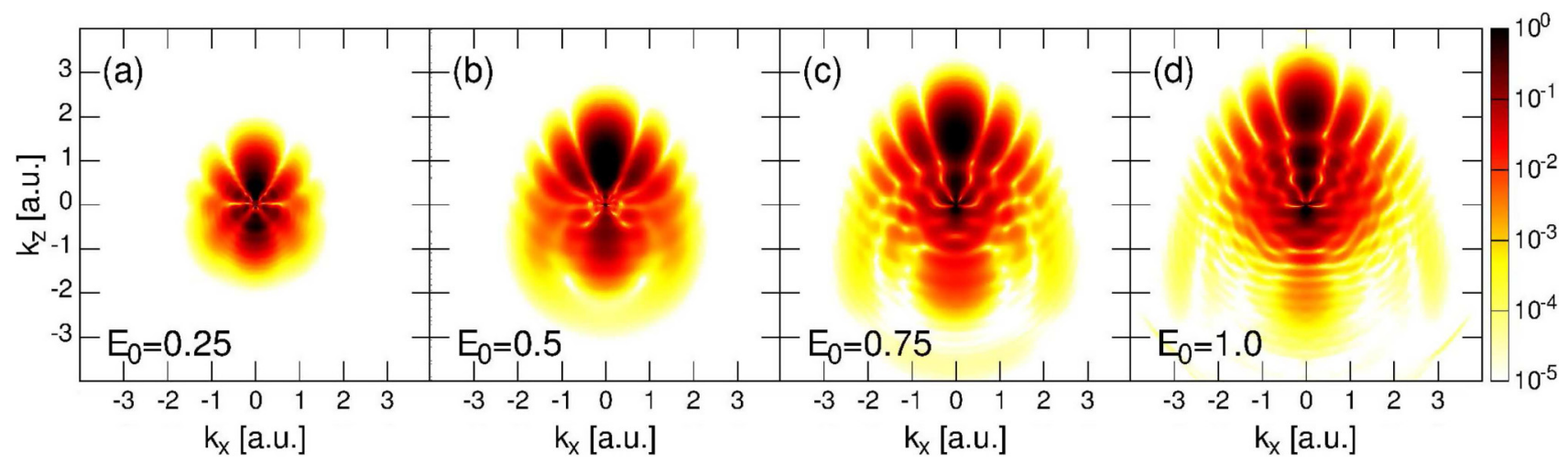

Fig. 10. Photoelectron spectra of the $\mathrm{H}_{2}^{+}$molecule (considered in the $R=2$ a.u. equilibrium configuration) calculated for different electric field amplitudes [(a) $E_{0}=0.25$ a.u., (b) $E_{0}=0.5$ a.u., (c) $E_{0}=0.75$ a.u., (d) $E_{0}=1$ a.u.].

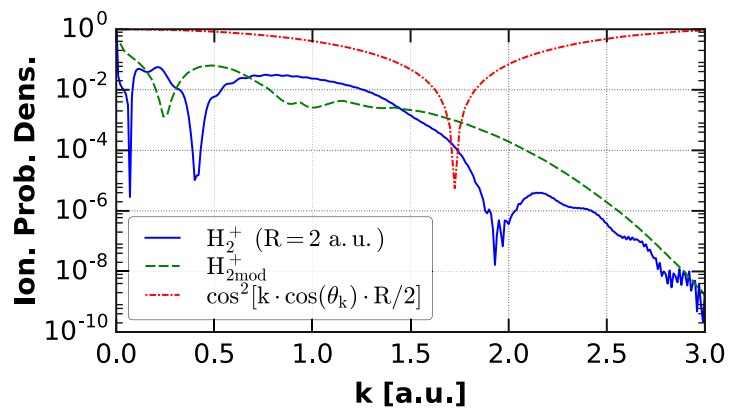

Fig. 11. PhES values along the first spatial interference minimum calculated for $\mathrm{H}_{2}^{+}$and for the $\mathrm{H}_{2 \bmod 1}^{+}$model plotted next to $\cos ^{2}(\boldsymbol{k} \cdot \boldsymbol{R} / 2) \equiv \cos ^{2}\left[k \cdot \cos \left(\theta_{k}\right) \cdot R / 2\right]$ on logarithmic scale.

photoelectron holograms is shown in Figure 10. It can be observed, that by increasing the amplitude the density of the photoelectron hologram (i.e., the number of radial interference extrema) also increases without changing its main features. This behavior is in agreement with our observations made in the case of atomic targets [10], and can be explained within the framework of the simple two-path model $[4,10]$ : with the increase in the field amplitude $\left(E_{0}\right)$ the $z_{0}$ parameter (the maximum distance reached by the electron trajectory before the recollision) also increases, which in turn induces a larger phase difference between the direct and indirect trajectory. This larger phase difference will change more rapidly with the change in the electron's asymptotic parameters leading to a denser interference pattern.

As it was argued before, in the photoelectron spectra the two-center interference minima (see the horizontal minima in Fig. 8) are not observable directly. However, when the $\mathrm{H}_{2}^{+}$and model photoelectron spectra for $R=2$ a.u. are compared along the first spatial interference minima. (observable around the $\theta_{k}=\pi / 8$ electron ejection angle in Fig. 5b) significant differences are observed. Figure 11 shows the depth of the spatial interference minimum as a function of the electron ejection momentum for the $\mathrm{H}_{2}^{+}$target and for its models as well. The high momentum $(k>1.5$ a.u. $)$ minimum observable only in the $\mathrm{H}_{2}^{+}$ curve is located closely to the two-center interference min- imum predicted by the simple model of Nagy et al. [55], where the two-center interference pattern is proportional to $\sim \cos ^{2}\left(\frac{\boldsymbol{k} \cdot \boldsymbol{R}}{2}\right)$. The shift in the location of the $\mathrm{H}_{2}^{+}$minimum appears due to the nonzero vector potential in the time moment when the continuum EWP showing the deep minimum was created. The minima observable for both the $\mathrm{H}_{2}^{+}$and its model at low electron momentum values are the result of the interference between the EWPs created during the second and third half cycle of the laser field [13]. This minimum is observable only for the $R=$ 2 a.u. internuclear distance at several field strengths and its position moves around the minimum of the $\cos ^{2}\left(\frac{\boldsymbol{k} \cdot \boldsymbol{R}}{2}\right)$ curve $^{2}$ as the field strength is changed. This change in the minimum's position might be attributed to different vector potentials of the driving field and also to the recollision of the returning EWP, however, despite our best effort, based on these effects we did not succeed to construct a model which reliably describes the movement of the minimum. The minimum appears in case of $R=1 \mathrm{a} . u$. for $k>3.5$ a.u., momentum for which the transition probability is very small, and the effect is irrelevant. For $R=4$ a.u. this effect, i.e., the two-center interference minimum, was not observed, most probably because the amplitude of the scattered EWPs on the two nuclei are very different due to the large internuclear distance. We have to emphasize that the main feature of the obtained hologram is governed by the interference between the direct and scattered EWPs, and not the interference between waves scattered on the different nuclei.

\section{Conclusions}

We have developed and presented an accurate theoretical tool based on the numerical solution of the timedependent Schrödinger equation (TDSE), which allows us to study the interaction between few-cycle (ultrashort) XUV laser pulses and the $\mathrm{H}_{2}^{+}$molecule. Using this tool we analyzed the photoelectron spectra (PhES) of

\footnotetext{
2 The observed minimum compared to the simple model minimum is located at a higher momentum value for $E_{0}=0.5$ a.u., at lower momentum value for $E_{0}=0.75$ a.u., while for $E_{0}=1.0$ a.u. they nearly coincide.
} 
$\mathrm{H}_{2}^{+}$calculated for different internuclear separations. The accurate PhES and photoelectron holograms (i.e., interference patterns created in the PhES by the interference between the scattered and reference electronic wave packets) were obtained after performing rigorous convergence tests. Beside the $\mathrm{H}_{2}^{+}$, we also considered one-center model systems $\left(\mathrm{H}_{2 \bmod 1}^{+}\right.$and $\left.\mathrm{H}_{2 \bmod 2}^{+}\right)$with the same ionization energy and long range potential as $\mathrm{H}_{2}^{+}$. These conditions ensured that during the interaction with the same XUV pulse it produced similar scattered and reference EWP trajectories as the $\mathrm{H}_{2}^{+}$target.

As expected, we obtained roughly similar PhES for both types of targets, and we have shown that the differences between the $\mathrm{H}_{2}^{+}$and model patterns can be directly attributed to the differences in the parent ions' potential that the signal electron meets along the scattering path during the recollision event: i.e., deeper the potential along the returning path, the higher the density of the minima locations. We have compared the obtained holograms for the $\mathrm{H}_{2}^{+}$and two spherically symmetric models. The two models lead to similar patterns, while clear differences were observed for the molecular potential relative to the central potentials. These differences may be directly attributed to the two-center character of the target. The observed differences in the forward-scattering holograms $\left(\theta_{k}<90^{\circ}\right)$ are small, which makes their experimental observation rather difficult, however these differences are significantly larger for the backward scattering holograms $\left(\theta_{k}>90^{\circ}\right)$. These larger differences are easier to measure in an experimental setup, thus the backward scattering holograms appears to be a more suitable candidate for structure analysis tool. The results presented here are not suitable to study in details the backward photoelectron hologram since the PhES is dominated by the forward scattering hologram. In order to make their study easier the parameters of the driving laser field should be changed in such way that the intensity of the backward scattering hologram becomes larger.

The direct fingerprint of the molecular structure, the two-center interference, was not observed here because of the complex superposition of the electron waves (direct and scattered) ejected at different time moments, which limits the direct applicability of the photoelectron holography in imaging molecules. However, by changing the parameters of the driving laser field, we can restrain the creation of the continuum EWPs to the immediate vicinity of each half cycle's peak, and by doing so we can eliminate the wave packet interference which smears out the two-center interference.

The investigation of the molecular photoelectron holograms in the above outlined laser pulse regimes will be the subject of a future work.

Furthermore, we have shown that the locations of the PhES minima obtained for the molecule changed as we modified the internuclear separation $R$. As we increased $R$ we obtained a denser interference pattern, which is caused by the interplay between two distinct factors. Firstly, with the increase in $R$ the ionization potential of the emitting molecule becomes lower, implicitly meaning a higher initial velocity of the ionized electron, which will travel a longer distance from the parent molecule (i.e., higher $z_{0}$ parameter before returning), and which will result in a higher minimum density in the hologram. Secondly, this effect is weakened by the fact that the returning electron will meet a shallower binding potential as the internuclear distance is increased, resulting in a decrease in the hologram density.

Open access funding provided by MTA Wigner Research Centre for Physics (MTA Wigner FK, MTA EK). GZsK gratefully acknowledges financial support from the Romanian Financing Authority UEFISCDI project ELI-NP 03-ELI/2016 (ProPW), and funding from the National Research, Development and Innovation Office of Hungary (grant VEKOP-2.3.2-16-201700015). SB was supported by a mobility grant of the Romanian Ministry of Research and Innovation, CNCS-UEFISCDI, project number MC-2019-1713, within PNCDI III. TA's work was supported by the EU-funded Hungarian grant No. EFOP3.6.2-16-2017-00005. The ELI-ALPS project (GINOP-2.3.615-2015-00001) is supported by the European Union and co-financed by the European Regional Development Fund.

\section{Author contribution statement}

All the authors were involved in the preparation of the manuscript. All the authors have discussed, read and approved the manuscript.

Publisher's Note The EPJ Publishers remain neutral with regard to jurisdictional claims in published maps and institutional affiliations.

Open Access This is an open access article distributed under the terms of the Creative Commons Attribution License (https://creativecommons.org/licenses/by/4.0/), which permits unrestricted use, distribution, and reproduction in any medium, provided the original work is properly cited.

\section{References}

1. R.J. Collier, C.B. Burckhardt, L.H. Lin, Optical Holography (Academic Press, 1971)

2. D. Gabor, Nature 161, 777 (1948)

3. M.E. Haine, T. Mulvey, J. Opt. Soc. Am. 42, 763 (1952)

4. Y. Huismans, A. Rouzée, A. Gijsbertsen, J.H. Jungmann, A.S. Smolkowska, P.S.W.M. Logman, F. Lépine, C. Cauchy, S. Zamith, T. Marchenko, J.M. Bakker, G. Berden, B. Redlich, A.F.G. van der Meer, H.G. Muller, W. Vermin, K.J. Schafer, M. Spanner, M.Y. Ivanov, O. Smirnova, D. Bauer, S.V. Popruzhenko, M.J.J. Vrakking, Science 331, 61 (2011)

5. X.-B. Bian, Y. Huismans, O. Smirnova, K.-J. Yuan, M.J.J. Vrakking, D. Bandrauk, Phys. Rev. A 84, 043420 (2011)

6. T. Marchenko, Y. Huismans, K.J. Schafer, M.J.J. Vrakking, Phys. Rev. A 84, 053427 (2011)

7. D.G. Arbó, E. Persson, J. Burgdörfer, Phys. Rev. A 74, 063408 (2006)

8. Y. Huismans, A. Gijsbertsen, A.S. Smolkowska, J.H. Jungmann, A. Rouzée, P.S.W.M. Logman, F. Lépine, 
C. Cauchy, S. Zamith, T. Marchenko, J.M. Bakker, G. Berden, B. Redlich, A.F.G. van der Meer, M.Y. Ivanov, T.-M. Yan, D. Bauer, O. Smirnova, M.J.J. Vrakking, Phys. Rev. Lett. 109, 013002 (2012)

9. D.D. Hickstein, P. Ranitovic, S. Witte, X.-M. Tong, Y. Huismans, P. Arpin, X. Zhou, K.E. Keister, C.W. Hogle, B. Zhang, C. Ding, P. Johnsson, N. Toshima, M.J.J. Vrakking, M.M. Murnane, H.C. Kapteyn, Phys. Rev. Lett. 109, 073004 (2012)

10. S. Borbély, A. Tóth, K. Tőkési, L. Nagy, Phys. Rev. A 87, 013405 (2013)

11. G. Porat, G. Alon, S. Rozen, O. Pedatzur, M. Krger, D. Azoury, A. Natan, G. Orenstein, B.D. Bruner, M.J.J. Vrakking, N. Dudovich, Nat. Commun. 9, 2805 (2018)

12. H. Agueny, J.P. Hansen, Phys. Rev. A 98, 023414 (2018)

13. S. Borbély, A. Tóth, D.G. Arbó, K. Tőkési, L. Nagy, Phys. Rev. A 99, 013413 (2019)

14. S. López, D.G. Arbó, Eur. Phys. J. D 73, 28 (2019)

15. M. Okunishi, T. Morishita, G. Prümper, K. Shimada, C.D. Lin, S. Watanabe, K. Ueda, Phys. Rev. Lett. 100, 143001 (2008)

16. S. Micheau, Z. Chen, A.T. Le, J. Rauschenberger, M.F. Kling, C.D. Lin, Phys. Rev. Lett. 102, 073001 (2009)

17. J. Xu, Z. Chen, A.-T. Le, C.D. Lin, Phys. Rev. A 82 , 033403 (2010)

18. C.D. Lin, A.-T. Le, Z. Chen, T. Morishita, R. Lucchese, J. Phys. B: At. Mol. Opt. Phys. 43, 122001 (2010)

19. C.I. Blaga, J. Xu, A.D. DiChiara, E. Sistrunk, K. Zhang, P. Agostini, T.A. Miller, L.F. DiMauro, C.D. Lin, Nature 483, 194 (2012)

20. S. Borbély, A. Tóth, K. Tőkési, L. Nagy, Phys. Scr. 2013, 014066 (2013)

21. A. Tóth, S. Borbély, K. Tőkési, L. Nagy, Eur. Phys. J. D 68, 339 (2014)

22. A. Palacios, J.L. Sanz-Vicario, F. Martín, J. Phys. B: At. Mol. Opt. Phys. 48, 242001 (2015)

23. R.E.F. Silva, F. Catoire, P. Rivière, H. Bachau, F. Martín, Phys. Rev. Lett. 110, 113001 (2013)

24. Y.V. Vanne, A. Saenz, J. Mod. Opt. 55, 2665 (2008)

25. X.-B. Bian, A.D. Bandrauk, Phys. Rev. Lett. 108, 263003 (2012)

26. M. Meckel, A. Staudte, S. Patchkovskii, D.M. Villeneuve, P.B. Corkum, R. Doerner, M. Spanner, Nat. Phys. 10, 594 (2014)

27. X.-B. Bian, A.D. Bandrauk, Phys. Rev. A 89, 033423 (2014)

28. K.-J. Yuan, X.-B. Bian, A.D. Bandrauk, Phys. Rev. A 90, 023407 (2014)
29. W. Yang, Z. Sheng, X. Feng, M. Wu, Z. Chen, X. Song, Opt. Express 22, 2519 (2014)

30. L. Chen, C. Huang, X. Zhu, P. Lan, P. Lu, Opt. Express 22, 20421 (2014)

31. M. Li, X. Sun, X. Xie, Y. Shao, Y. Deng, C. Wu, Q. Gong, Y. Liu, Sci. Rep. 5, 8519 (2015)

32. M. Haertelt, X.-B. Bian, M. Spanner, A. Staudte, P.B. Corkum, Phys. Rev. Lett. 116, 133001 (2016)

33. Y. Li, Y. Zhou, M. He, M. Li, P. Lan, P. Lu, Phys. Rev. A 94, $013422(2016)$

34. M. He, Y. Zhou, Y. Li, M. Li, P. Lu, Opt. Quantum Electron. 49, 232 (2017)

35. H. Miyagi, T. Morishita, S. Watanabe, Phys. Rev. A 85, $022708(2012)$

36. J.P. Grivet, J. Chem. Ed. 79, 127 (2002)

37. M. Vincke, D. Baye, J. Phys. B: At. Mol. Opt. Phys. 39, 2605-2618, (2006)

38. D. Baye, J. Phys. A: Math. Theor. 44, 395204 (2011)

39. Y.-J. Jin, X.-M. Tong, N. Toshima, Comput. Phys. Commun. 182, 146 (2011)

40. H. Liang, X.-R. Xiao, Q. Gong, L.-Y. Peng, J. Phys. B: At. Mol. Opt. Phys. 50, 174002 (2017)

41. D.A. Telnov, S.I. Chu, Comput. Phys. Commun. 182, 18 (2011)

42. W. Miller Jr., A.V. Turbiner, J. Phys. A: Math. Theor. 47, 192002 (2014)

43. T.N. Rescigno, C.W. McCurdy, Phys. Rev. A 62, 032706 (2000)

44. A. Palacios, C.W. McCurdy, T.N. Rescigno, Phys. Rev. A 76, 043420 (2007)

45. D. Dundas, Phys. Rev. A 65, 023408 (2002)

46. L.-Y. Peng, D. Dundas, J.F. McCann, K.T. Taylor, I.D. Williams, J. Phys. B: At. Mol. Opt. Phys 36, 295 (2003)

47. G.Zs. Kiss, S. Borbély, L. Nagy, AIP Conf. Proc. 1916, 20010 (2017)

48. V. Hernandez, J.E. Roman, V. Vidal, ACM Trans. Math. Softw. 31, 351 (2005)

49. C. Lanczos, J. Res. Nat. Bureau Stand. 45, 255 (1950)

50. M. Hiyama, H. Nakamura, Comput. Phys. Commun. 103, 209 (1997)

51. K.-J. Yuan, H. Lu, A.D. Bandrauk, Phys. Rev. A 83, 043418 (2011)

52. K.-J. Yuan, H. Lu, A.D. Bandrauk, Struct. Chem. 28, 12971309 (2017)

53. L. Czipa, L. Nagy, Phys. Rev. A 95, 062709 (2017)

54. S. Selst $\varnothing$, J.F. McCann, M. Førre, J.P. Hansen, L.B. Madsen, Phys. Rev. A 73, 033407 (2006)

55. L. Nagy, S. Borbély, K. Póra, Phys. Lett. A 327, 481 (2004) 